Preprint submitted to Remote Sensing of Environment

(c) 2018. This manuscript version is made available under the CC-BY-NC-ND 4.0 license

http://creativecommons.org/licenses/by-nc-nd/4.0/

Final version at https://doi.org/10.1016/j.rse.2018.08.034

\title{
Vegetation and soil moisture inversion from SAR closure phases: first experiments and results
}

\author{
Francesco De Zan, Giorgio Gomba \\ German Aerospace Center (DLR), Oberpfaffenhofen, Germany
}

\begin{abstract}
The inversion of soil moisture from Synthetic Aperture Radar (SAR) closure phases is intrinsically plagued by ambiguities that affect the moisture order. This work shows a characterization of the ambiguities and a way to solve for them with the help of interferometric coherence. This allows to properly constrain the inversion and to retrieve the moisture signal. A data set of ALOS-2/PALSAR-2 L-band images is used as an example of successful inversion at the scene level, with sub-kilometer resolution. The results are validated with soil moisture products based on ASCAT and show a high degree of correlation. The raw moisture derived by the algorithm could be immediately used to correct SAR interferometric phases; however, for applications that need absolute moisture levels, a calibration step is likely necessary. Unexpectedly, a good performance was observed over forested areas, which suggests a sensitivity of closure phases to tree moisture; at the same time, over pastures and agricultural fields the closure phase signal was found relatively weak. Additional research is needed to evaluate the applicability of the same measurements principle to shorter wavelengths and exploitation of potential synergies with backscatter and polarimetric information.
\end{abstract}

Keywords: soil moisture, closure phase, SAR interferometry

\section{Introduction}

Soil moisture is a key variable in modeling the water cycle, energy and carbon fluxes and therefore relevant for many disciplines including hydrology, meteorology, and climatology (Ochsner et al. (2013); Wagner et al. (2012)). Whereas moisture probes give precise point measurements, the high spatial variability of the moisture signal limits the usefulness of single probes or even sensor networks for characterizing a given area (Peng et al. (2017); Crow et al. (2012)). Remote sensing techniques are useful in sensing the moisture field over large areas, with their limitations: coarse spatial resolution (e.g. $>10 \mathrm{~km}$ ), sparse temporal sampling (e.g. a few days), sensitivity only to the first centimeters of soil (i.e. no root zone moisture) (Mohanty et al. (2017)). Currently, the most successful wide-area retrieval concepts belong to the field of microwave remote sensing, active and pas- sive (Peng \& Loew (2017); Das \& Paul (2015); Kornelsen \& Coulibaly (2013)).

Both active and passive techniques often require compensation of unwanted influences related to the vegetation cycle and surface roughness (Brocca et al. (2011); Wagner et al. (1999)). Products derived from synthetic aperture radar backscatter cannot fully exploit the high resolution of SAR images: extensive spatial averaging is typically needed in order to counter the instability of surface roughness (Thoma et al. (2008)).

This paper presents a novel moisture measurement concept based on SAR closure phases. This concept has the potential to offer moisture products with fine spatial resolution (e.g. $500 \mathrm{~m}$ or better), which is one unmet need identified in Peng \& Loew (2017), the other being high temporal resolution. Considering the three interferograms $I_{l, m}, I_{m, n}, I_{n, l}$ generated with three images $l, m, n$ and averaged spatially, the 
closure phase is simply the phase of the cyclic product of the interferograms:

$$
\Phi_{l, m, n}=\arg \left(I_{l, m} I_{m, n} I_{n, l}\right) .
$$

Closure phases are interferometric observables whose potential in SAR has not been entirely explored yet (De Zan et al. (2015)). It has been known for a few years that interferometric and closure phases carry information on soil moisture (Morrison et al. (2011); De Zan et al. (2014); Zwieback et al. (2015)), however the retrieval of moisture levels from closure phases has not shown any progress (Zwieback et al. (2017)). On the other hand, there would be obvious advantages in using closure phases instead of interferometric phases for moisture inversion: closure phases are immune to all simple propagative effects like target displacement, delays in atmospheric propagation, topographic effects, i.e. the usual contributors to the interferometric phase.

The approach proposed in this contribution could complement existing methods (radiometric or scatterometric) for soil moisture retrieval. However, with this work we do not claim to introduce an operational, efficient, and validated technique for soil moisture retrieval. We report first experiments with L-band data, which we selected considering the coherence advantage and the fact that closure phases are larger in Lband compared to higher frequencies. Our first results are promising; however, the only L-band spaceborne SAR sensor today is PALSAR-2 onboard ALOS-2, and for any operational soil moisture applications its spatial and temporal sampling is likely insufficient. However, more satellite L-band SAR's are being launched, and we should prepare today for future opportunities.

The physical modelling and understanding is also not complete: in the data set we examined, for example, the scale of the closure phase signal is rather weak over the (relatively small) areas of pasture and rice fields. Surprisingly, we were able to perform consistent inversions over large forested areas, where the closure signal is very strong. All this shows the need for further understanding before an operational algorithm can be designed and the potential of closure phases can be fully harnessed.

Apart from the potential for soil moisture retrieval, the successful inversion of a moisture model will allow correcting the interferometric phases, both for single interferograms and for multi-image interferometric processing. These corrections are especially relevant for the lower frequency SAR's, since the effect of moisture variations roughly scales with the wave- length (Zwieback et al. (2017)).

In this publication we specifically address the presence of an ambiguity in the moisture model for closure phases: the same set of observed closure phases can be explained almost equally well by different sets of moisture levels. The ambiguity arises because the closure phases can only partially constrain the ordering of the acquisitions according to moisture levels. This is illustrated in Section 2. Once this ambiguity is tackled explicitly and correctly solved, the way is open for reliable moisture inversion (Section 3). Section 4 presents inversion results and a comparison with several available products. Successful inversion of moisture levels allows compensating moistureinduced contributions to interferometric phases (Section 5) thus improving traditional repeat-pass InSAR products like deformation monitoring of the Earth's crust.

\section{Ambiguities in closure phases models}

\subsection{Interferometric models for moisture}

We want to model the interferograms as a function of the moisture values in the two interfering acquisitions, indexed by $l$ and $m$. Our starting point is Eq. (11) in (De Zan et al. (2014)), which derives the expected value of the interferogram as a function of the two wavenumbers $\left(k_{l}\right.$ and $\left.k_{m}\right)$ and the scattering profile in the soil $f(z)$. We report it here for convenience:

$$
I_{l, m}=\int_{0}^{\infty} f(z) \exp \left(-j 2 k_{l} z\right)\left(\exp \left(-j 2 k_{m} z\right)\right)^{*} d z .
$$

The star indicates the complex conjugation operation.

The wavenumbers $k_{n}$ depend on the dielectric constant, which is a function of the moisture levels $\left(\theta_{n}\right.$, in the following) and soil type (Hallikainen et al. (1985); Bircher et al. (2016)). A good approximation is to take the formula for normal incidence (Morrison \& Bennett (2015); De Zan et al. (2014))

$$
k=\sqrt{\omega^{2} \mu \epsilon},
$$

where $\omega$ is the angular frequency, $\mu$ and $\epsilon=\epsilon(\theta)$ are respectively the dielectric permeability and permittivity.

Assuming an exponential scattering profile $f(z)=$ $\exp (-2 \alpha), \alpha>0$, the expected value of the interferogram is:

$$
I_{l, m}=\frac{1 / 2}{j\left(k_{l}-k_{m}^{*}\right)+\alpha} .
$$


If $\alpha=0$, the model is trivially the one of Eq. (12) in (De Zan et al. (2014)),

$$
I_{l, m}=\frac{1 / 2}{j\left(k_{l}-k_{m}^{*}\right)},
$$

and the scattering intensity at different depths is governed solely by the dielectric constant itself, or more precisely, by the imaginary part of the wavenumbers. Note that the interferogram in Eq. (5) is not normalized: normalization is straightforward and is necessary if one needs to compute interferometric coherences. The coherence decays approximately with moisture difference, as one can see in De Zan et al. (2014). The corresponding closure phase $\Phi_{l, m, n}$ is simply the phase of the cyclic triple product

$$
\Phi_{l, m, n}=\arg \left(\frac{1 / 2}{j\left(k_{l}-k_{m}^{*}\right)} \frac{1 / 2}{j\left(k_{m}-k_{n}^{*}\right)} \frac{1 / 2}{j\left(k_{n}-k_{l}^{*}\right)}\right) .
$$

It is useful to consider another specialization of Eq. (4), by discarding the imaginary part of the wavenumbers and setting necessarily $\alpha \neq 0$ :

$$
I_{l, m}=\frac{1 / 2}{j\left(k_{l}-k_{m}\right)+\alpha} .
$$

This case describes also the SAR tomographic setting (see Dall (2007), Eq.(9)), in which it is common to assume that the variations of the viewing angle do not affect the scattering profile. This model is useful to derive an approximation to the closure phase (De Zan et al. (2015))

$$
\begin{aligned}
\Phi_{l, m, n} & =\arg \left(I_{l, m} I_{m, n} I_{n, l}\right) \\
& \approx-\alpha^{-3}\left(k_{l}-k_{m}\right)\left(k_{m}-k_{n}\right)\left(k_{n}-k_{l}\right) .
\end{aligned}
$$

By further approximating $k_{l}-k_{m} \propto \theta_{l}-\theta_{m}$, i.e. the soil moisture difference, one can obtain a direct link between closure phases and moisture variations:

$$
\Phi_{l, m, n} \approx-\alpha^{-3}\left(\theta_{l}-\theta_{m}\right)\left(\theta_{m}-\theta_{n}\right)\left(\theta_{n}-\theta_{l}\right)
$$

where the proportionality parameter $\alpha$ will have to be properly adjusted.

\subsection{Ambiguities in sorting the acquisitions according to the moisture level}

Ambiguities in closure phase models block the way to the successful inversion of the parameters of interest, in this particular case the moisture level, as (Zwieback et al. (2017)) has clearly identified. In this section we will try to shed light on the character of

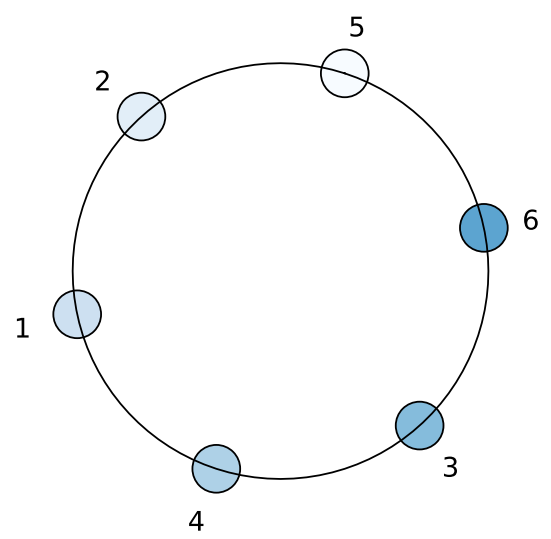

Figure 1. Closure-phase signs allow sorting acquisitions according to increasing moisture, but the result is wrapped. In the example, acquisitions 5 is the driest, 6 is the wettest, as indicated by the color.
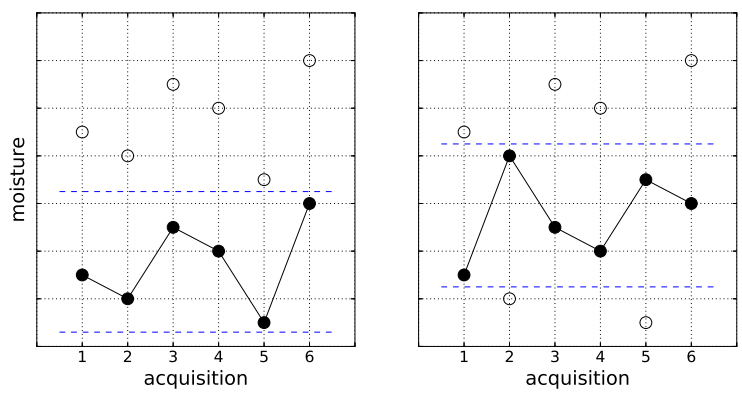

Figure 2. Left: one possible moisture evolution is displayed with filled circles. The open circles replicate the evolution to simulate the circular wrapping of the moisture ordering. Right: an alternative moisture ordering that would generate the same closure phase signs. It corresponds to a different selection of the ambiguity interval (dashed horizontal lines). Additional information is needed to select the correct solution.

the ambiguities. We are going to base our discussion on the signs of the closure phases, as we observed that ambiguities arise in the inversion when two or more moisture histories yield a set of closure phases which have the same signs. We start by considering the simplified model for closure phases given by Eq. (9). This model is not totally equivalent to the one of Eq. (6), however the signs of the closure phases are identical, as one can easily verify. The two models are therefore considered equivalent for our purpose, and the conclusions will be valid for both.

It is immediate to verify that cyclical permutations of the moisture ordering of three acquisitions will not change the sign of $\Phi_{l, m, n}$ (Zwieback et al. (2017)). The same extends to any number of acquisitions and any closure phase that can be generated with those acquisitions. Thus it follows that the signs of the closure phases can be exploited to sort the acquisitions according to the moisture level, up to a special kind of 
ambiguity: The signs of the closure phases are invariant to cyclic permutations of the moisture order. Fig. 1 illustrates an example of the ambiguous ordering of six acquisitions. This ambiguity means that there are many indistinguishable sorting possibilities: for instance, both orders $(5,2,1,4,3,6)$ and $(1,4,3,6,5,2)$ of increasing moisture are acceptable by looking just at the signs of the corresponding closure phases. Of course the different permutations correspond to totally different moisture trajectories in time as shown in Fig. 2.

The central problem is therefore recovering the right order among those allowed by the closure phase signs. This is equivalent to finding out the acquisition with the highest moisture level, or the lowest. In general, with $N$ acquisitions there are $N$ ambiguous solutions. One can think of different ways of resolving the ambiguity either using SAR observables or external data.

\subsection{Additional data are needed to solve the ambiguities}

External data (moisture data from other sensors, or from numerical weather models) could help identifying, for instance, the acquisition with the highest or the lowest moisture level. Nevertheless the availability, accuracy and spatial resolution of these measurements is not sufficient for our objectives. It is therefore necessary to extract this information directly from the SAR images.

Using SAR backscatter as a proxy for moisture is also a possibility (Wagner et al. (2007)). According to our limited experience, it is hard to reliably identify the wettest or the driest acquisition from the backscatter series. Note that this is a different application from reconstructing the moisture evolution from the backscatter, for which there exist operational algorithms. Our lack of success may also be explained by insufficient spatial mitigation of temporal roughness instability, or by seasonal variations in the backscatter not related to moisture changes. In any case, we soon abandoned this way when we found a viable solution based on the local interferometric coherence.

The local interferometric coherence is influenced by temporal decorrelation, volumetric decorrelation, ground changes, and also by soil moisture. For this reason, a direct estimation of the soil moisture from the coherence is probably impossible. Nevertheless, we think that the coherence can be used to unravel the ambiguity issue: our concept is to collect a list of rules that a wrong soil moisture ordering would break, therefore allowing us to reject it, or at least consider it as unlikely. For example, images with different levels of soil moisture should typically present lower coherence levels than images with similar moisture. At the same time one should not forget that measured coherence values can be lower than predicted from the inverted moisture, because of temporal decorrelation or other effects, but for sure they cannot be higher. The coherence compensated with the predicted moisture is expected to decrease with time. These considerations allow to evaluate the likelihood of any given solution of the ambiguity.

\section{Inversion algorithm}

Our objective is the recovery of the moisture changes that explain the closure phases. The input data of the inversion are therefore the observed closure phases $\hat{\Phi}_{l, m, n}$, whereas the moisture levels are the unknown variables to be estimated. We collect them in the vector $\underline{\theta}$. The inverse problem is solved by minimizing a function that represents how well the data predicted by the moisture model fit the observations. We simply took the mean square difference between predicted and observed data:

$$
E(\underline{\theta})=\sum_{l, m, n}\left(\hat{\Phi}_{l, m, n}-\Phi_{l, m, n}(\underline{\theta})\right)^{2} .
$$

Due to the non-linearity of the problem the cost function presents many local minima: a nonlinear optimization method would then, in theory, be necessary. However, the global minimum of the cost function is not necessarily associated with the correct soil moisture sequence because of the ambiguities in the model and of the data noise. For this reason, global optimization algorithms are alone not sufficient to find the correct solution of the problem.

The method we developed to estimate the soil moisture consists of three parts:

(I) For each data pixel, we first establish in which sequence the acquisitions are connected in order to ensure monotonically increasing moisture values. That is, we establish the moisture order, subject to circular permutation ambiguity. The acquisitions are now arranged in a chain like in Fig. 1;

(II) In the second part, we determine which of the acquisitions presents the highest moisture level, we therefore solve the ambiguity and break the moisture circle at the correct point. This stage corresponds, for example, to choosing the solution on the left in Fig. 2;

(III) Finally, we recover the actual moisture levels by a global minimization of the cost function while 
constraining the variables to keep the order established in the latter step. The next sections will enlighten these procedures more in detail. Figure 3 shows a schematic representation of the whole procedure.

Among all possible closure phase combinations, only a subset contains independent data. For $N$ images, the number of independent closure phases is $(N-1) \cdot(N-2) / 2$. In our inversions we always use at least as many closure phases as the number of independent ones. At the same time the number of unknowns is only $N-1$ since we do not seem to have enough sensitivity to estimate the starting value of the moisture sequence and we fix it arbitrarily to a reasonable value. With increasing number of acquisitions, the number of independent data outgrows quickly the number of unknowns, thus making the result more reliable. For example, with 4 images there are 3 independent closure phases and 3 unknowns, with 12 images there are 55 independent closure phases and 11 unknowns.

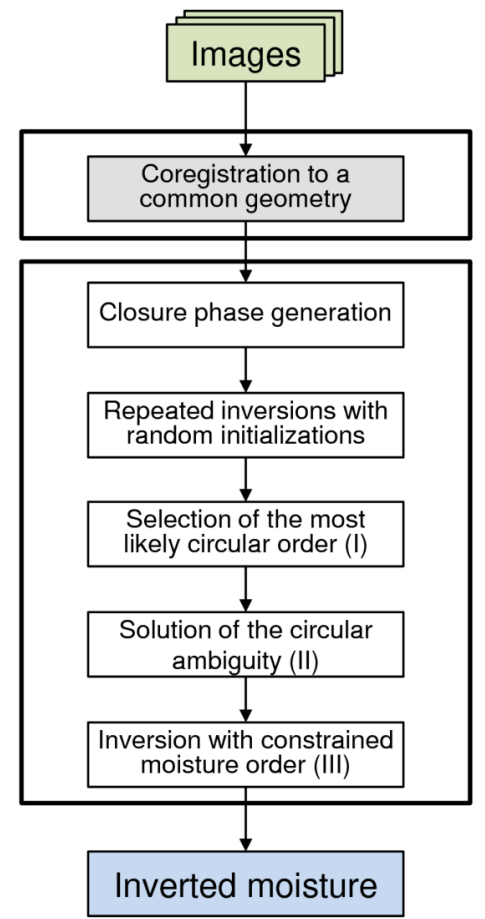

Figure 3. Block diagram of the inversion algorithm.

\subsection{Collection of Ambiguous Solutions}

According to Section 2, due to the ambiguity of the closure phase problem, various moisture sequences can produce almost the same closure phases. For this reason, a global optimization will find multiple solutions with similar levels of mean square error that are circularly similar.

In our method we run multiple times a simple minimization algorithm (Powell's method) that can only find local minima, for each run we use a different random starting point in the moisture domain. This way, the solutions that are produced should have comparable small error and either be the correct one, or a circularly equivalent solution, or a different solution. If the phase noise is sufficiently low and the physical model is correct, we assume that the most frequent solutions and with lowest cost that are found belong to the same moisture chain, i.e. the correct circular ordering. The ambiguity has now to be solved, the images with the maximum and minimum moisture must be identified to open the chain and establish the final moisture order.

\subsection{Solution of the Ambiguity}

Based on the premises expounded in Sect. 2.2, we developed a first simple algorithm which has shown to provide acceptable results with our test cases; it might have to be improved for other scenarios. We base our rule on the expectation that the observed coherence should be lower for acquisitions with different soil moisture than for acquisitions with similar moisture. Therefore, images which are distant in the (correct) moisture order, thus possibly having the most different moisture values, should present the lowest coherence levels among all. On the contrary, images which are close in the moisture order should present the highest levels of coherence. We exploit this to separate the wettest from the driest image. Each image is tested for the position of the wettest element in the chain: Let $B_{i}$ be the set of measured coherence values for pairs separated by $i$ steps in the order under test. For each bin we select the maximum coherence $m_{i}=\max \left(B_{i}\right)$ and fit a line as a function of the bin (i.e. through the points $\left.\left(i, m_{i}\right)\right)$. The image which gives the steepest downward line is selected as the correct one. The choice of picking the maximum coherence instead of the average needs some explanation. Note that for small moisture differences there is no guarantee to measure high coherence as other factors might come into play and degrade the coherence. However there are typically many pairs with small moisture difference in a data set, and we require only the maximum of their coherences to be high. For large moisture differences, instead, there is no doubt that we should always observe low coherences. 

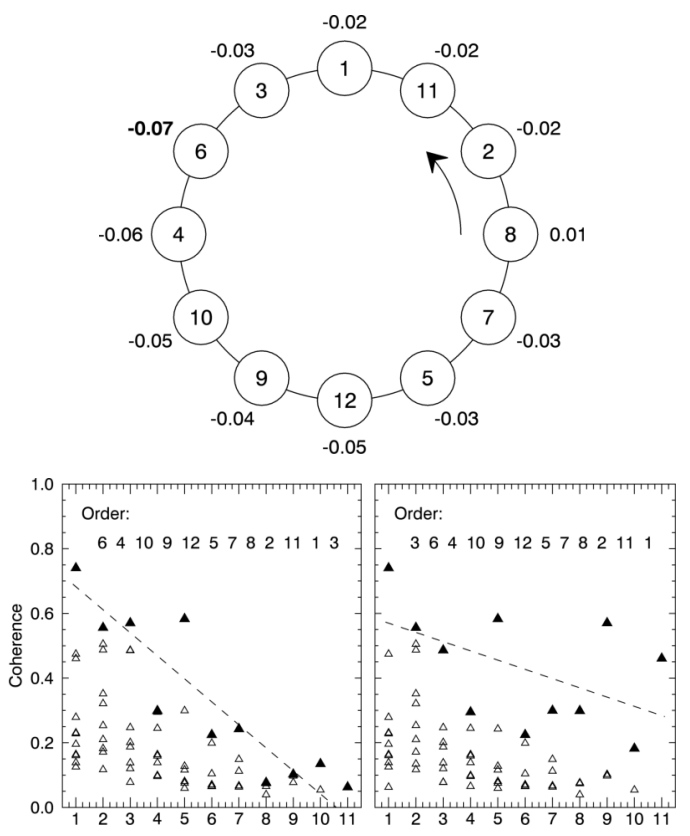

Figure 4. Example of ambiguity solution. At the top, the images chain: $(1,3,6,4,10,9,12,5,7,8,2,11)$ in decreasing order as indicated by the arrow; the maximum value is still unknown. In the bottom, coherence plots for two maximum candidates: 6 and 3. Each triangle is the pixel's coherence of an interferogram, its abscissa is the distance between the two images of the interferogram in the supposed order. The dashed line is fitted to the maximum coherences of each distance: the black triangles. The outer numbers in the top chain are the slope of the fitted lines. The steepest line is formed when the image 6 is selected as the maximum one, as in the left plot.

Figure 4 illustrates this procedure with a realdata example. Let us suppose that, with 12 images, the selected chain in decreasing order is: $(1,3,6,4,10,9,12,5,7,8,2,11)$, any circular shift of this chain might represent the correct order of moisture. To decide whether, for instance, it is more likely for number 6 or number 3 to be wettest date, we show the two respective coherence plots. The abscissa is the distance in the order: if we are plotting the pixel's coherence of the interferogram between images 6 and 8 when the supposed maximum is 6 (i.e. in the left plot), the distance is 7 . The gradient of the line fitting the maximum coherences (black triangles) is indicated close to the respective chain maximum in the top part of the figure. It becomes clear that the number 6 is more likely to be the right choice, since it corresponds to the steepest downward line. In fact, if we select the number 6 as wettest, we see that the principle that says that images with similar moisture have high coherence and different ones low coherence is honored by and large.

\subsection{Constrained Inversion}

Once the order has been established a constrained inversion can be performed, fixing the order of the moisture to the selected one. We use a simple constrained optimizer, which runs multiple times with different random starting points to achieve a global search. Finally, the moisture solution with the lowest cost is selected. The moisture of the first image is kept constant and not inverted, as the sensitivity of the closure phase to a moisture offset is not sufficient to recover it.
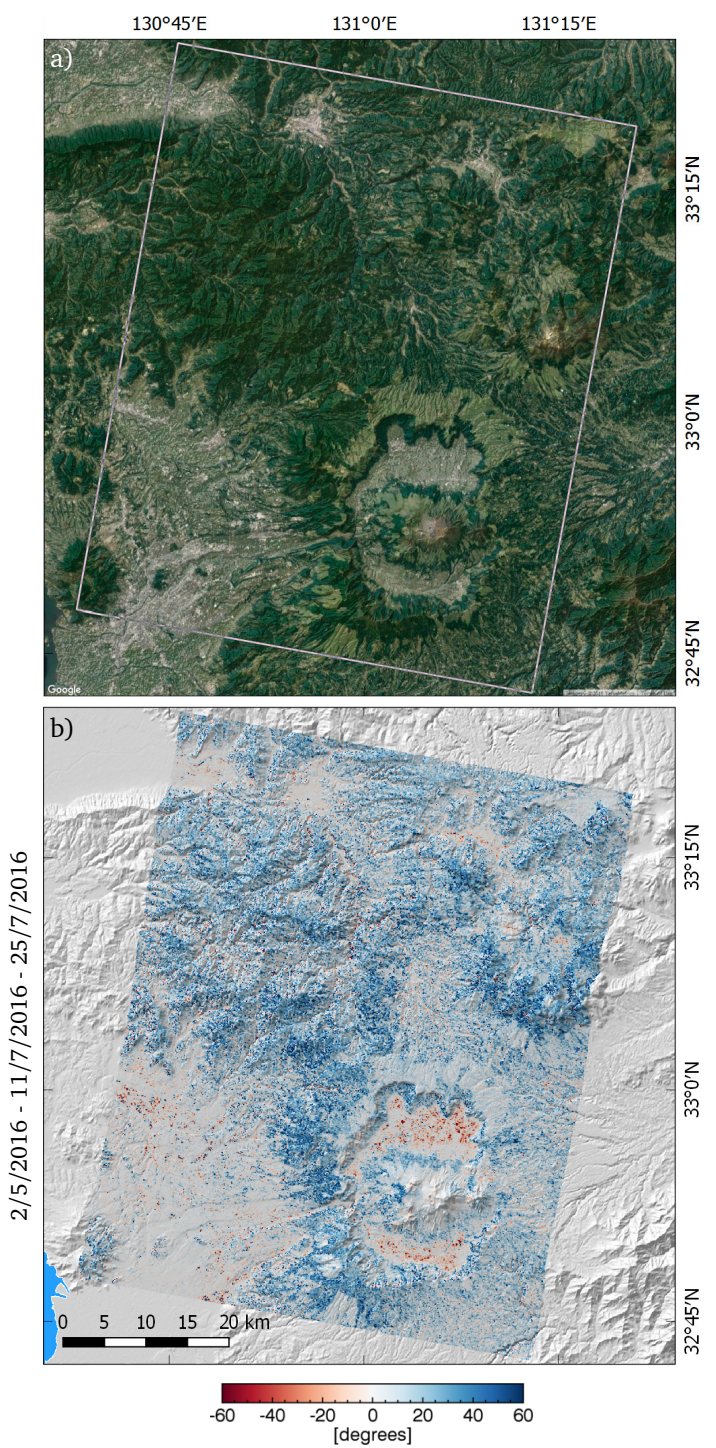

Figure 5. Area of Kumamoto, Japan: optical satellite photo from Google maps service (a) and geocoded closure phase (b) with exaggerated hillshading. 


\section{Results and Validation}

We experimented with an ALOS-2/PALSAR-2 data set consisting of 12 images, acquired in HH polarization from March to November, 2016 over the area of Kumamoto, Japan.

We tested two forward models: the physical one of Equation (6) with Hallikainen dielectric model (Hallikainen et al. (1985)), and the simplified one of Equation (9). The results are quite similar but the physical model can better fit the data: its mean square error is generally lower than the simplified model's one. We are therefore presenting the physical model inversion results.

We used several products to compare with our results: the surface soil moisture product based on ASCAT scatterometer measurements provided by the EUMETSAT's H SAF project, the soil moisture product from ESA Climate Change Initiative (ESA CCI v04.2 COMBINED), the ERA5 reanalysis product by ECMWF and the surface soil moisture provided by the SMAP mission (Level-4 Global 3-hourly 9 km EASE-Grid Analysis Update). One must say that SMAP does not provide level-2 radiometric measurements over our area of interest because of radio-frequency interference.

\subsection{Kumamoto}

Figure 5a shows the extent of the SAR image and ground elevation of the Kumamoto area derived from the SRTM DEM. As it can be seen from the optical satellite photo of the area in Figure $5 \mathrm{a}$, the ground coverage includes coniferous forests, grasslands, rice cultures, and cities. Figure $5 \mathrm{~b}$ is an example of geocoded closure phase, which can be compared to the ground cover. Figure 6 shows other phase examples, it can be easily noted that forested areas often present high closure phase values, different from grassland and urban areas.

The SAR images were acquired in stripmap mode with $80 \mathrm{MHz}$ range bandwidth yielding a range resolution of about 3 meters and an azimuth resolution of about 3 meters. Interferometric processing used a 90 by 90 pixels multilooking window. Finally, after the inversion, a median filter with a square window of four pixels was applied, producing a final ground resolution of about 540 meters in range and azimuth. This resolution is limited by our ability to recover stable moisture ordering: there are chances that it can still be improved. The mean coherence of the 66 interferograms spans from 0.11 to 0.67 with an average of 0.3 .

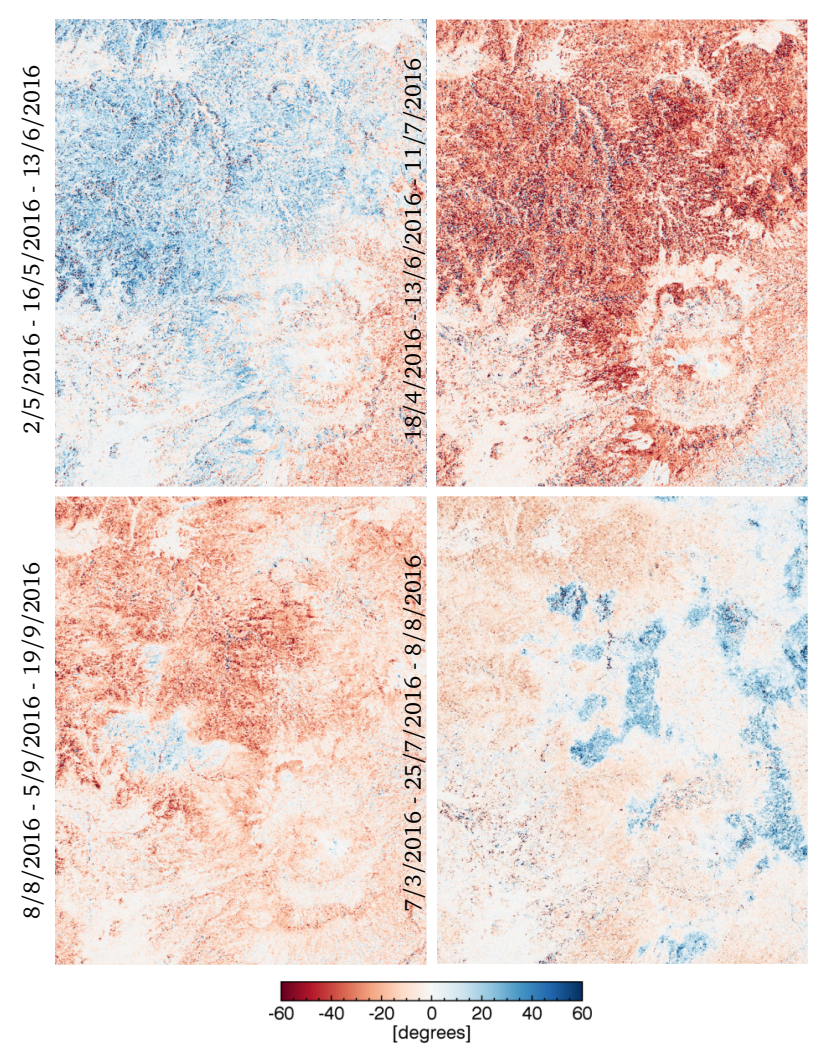

Figure 6. Example of different closure phases in the area of Kumamoto, Japan. A good correlation with the ground coverage is clearly visible, forested areas generally present larger phase levels. The closure phase is also higher in zones where a rain event occurred (bottom right image, blue areas).

From 12 images, 55 independent closure phases have been produced and used for the inversion. The results are presented in Figure 7. Moisture values for the first SAR image are missing as it has been used as a reference for the other dates. The bottom right picture in Figure 7 indicates the index of the image with the highest moisture level, according to the algorithm outcome. It can be seen that, in forested areas, the maximum is mostly either in the sixth of tenth image, but, randomly, also in other images. These local inhomogeneities might be due to incorrect estimation of the maxima. The presence of salt and pepper noise in the estimated moisture likely indicates the picking of a wrong maximum.

The offset and the scale of the results were calibrated to best fit the ASCAT data: the closure phase is only little sensitive to offset changes, and errors in the assumed porosity or mismodeling on our side can influence the scaling factor.

Unfortunately we were not able to find moisture probes in the area, the validation is therefore only 


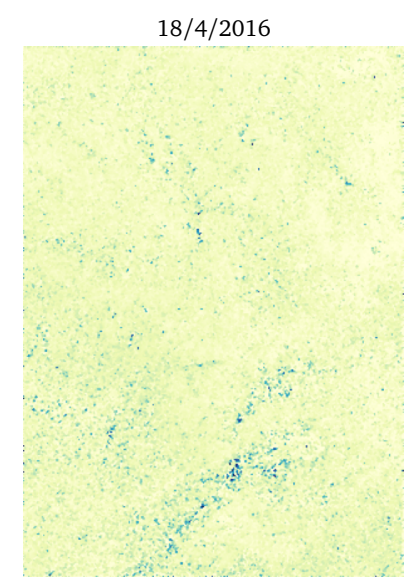

$11 / 7 / 2016$

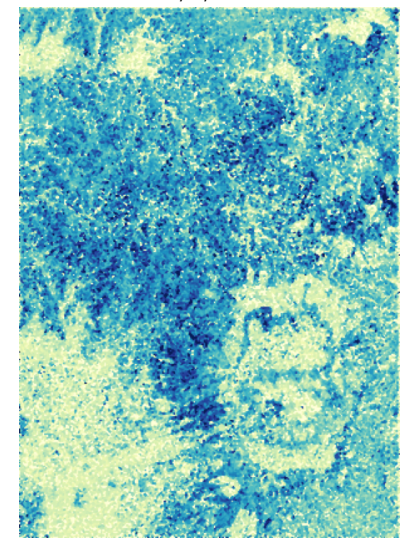

$19 / 9 / 2016$

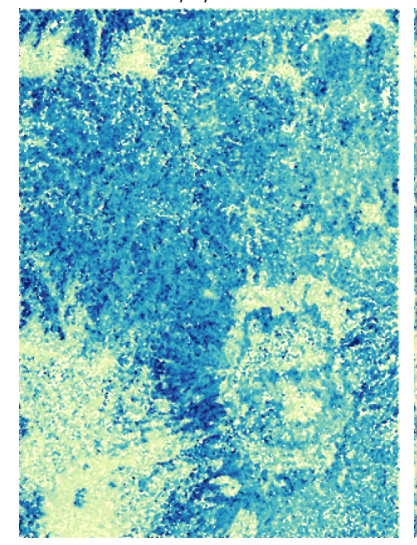

$2 / 5 / 2016$

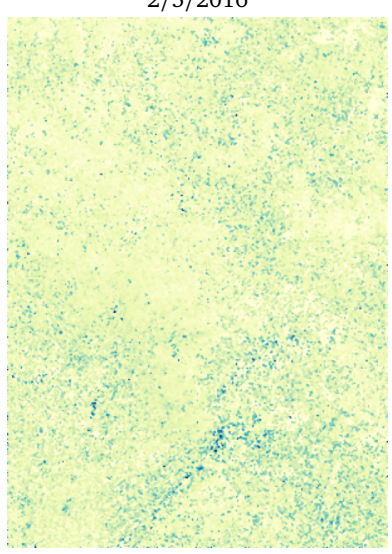

$25 / 7 / 2016$

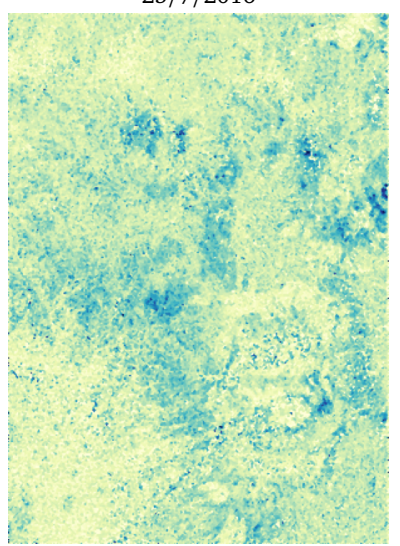

$3 / 10 / 2016$

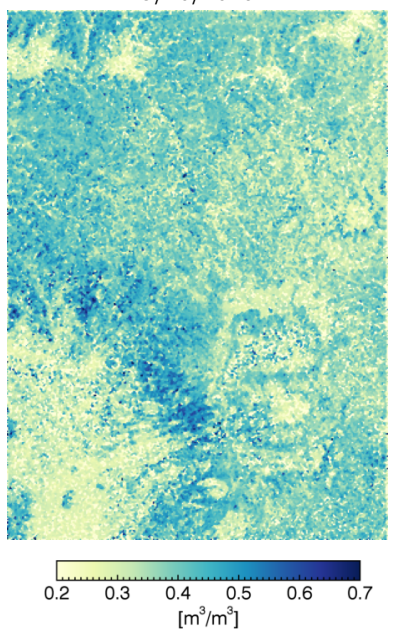

$16 / 5 / 2016$

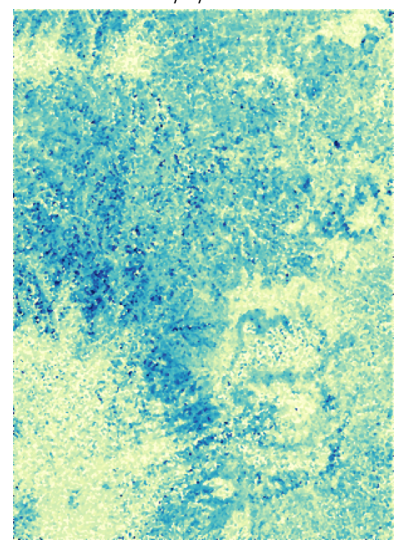

$8 / 8 / 2016$

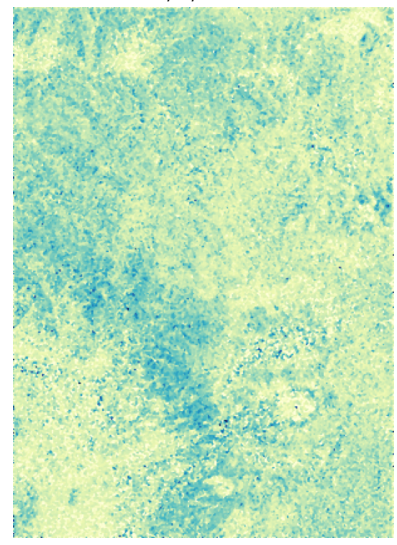

$31 / 10 / 2016$

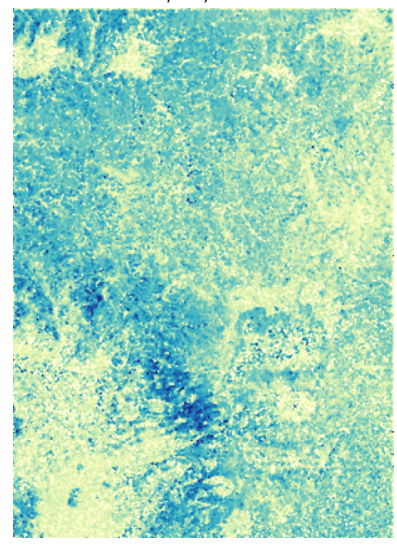

棈.

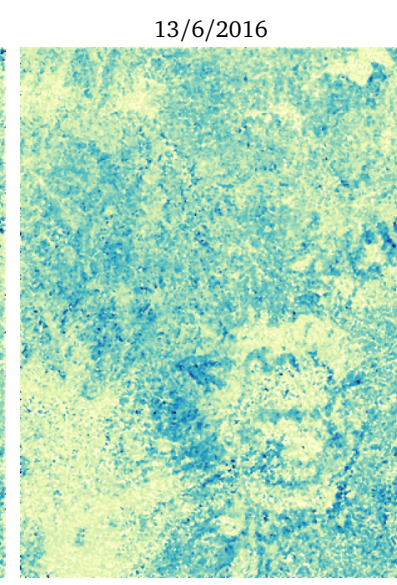

$5 / 9 / 2016$
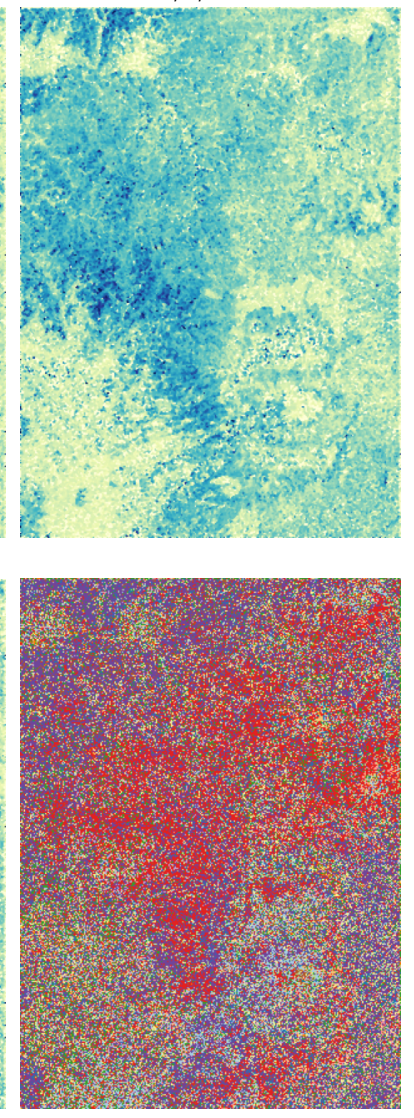

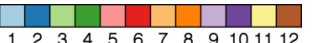

Figure 7. Inverted moisture values of the Kumamoto area, linearly scaled to fit ASCAT data. Bottom right: index of the image with the highest moisture level.

done with remote sensing and global reanalysis products. Figure 8 shows a good agreement especially between the moisture evolution from ALOS-2 closure phases and the ASCAT product. Both are based on active sensors, but the measurement concepts are rather different, one being incoherent and the other coherent. The correlation with ESA CCI, ECMWF and SMAP is less pronounced but some common features are recognizable. One cannot avoid noticing the discrepancies among the different products. 


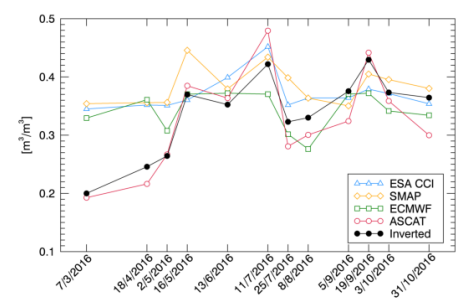

Figure 8. Comparison of Kumamoto inversion results with other available moisture products. The ASCAT product has been scaled to volumetric moisture assuming a porosity of $50 \%$.

We have analysed our inversion results for different classes of land cover: crop fields, grassland and forest. The results are reported in Figure 9. The forest class presents the largest excursion, in line with the fact that the observed closure phases are larger in magnitude over forests. It appears that a single scaling law is not enough to yield comparable soil moisture evolutions for all land covers. In our case the image is dominated by the forest and the scaling would therefore be appropriate only for this class.

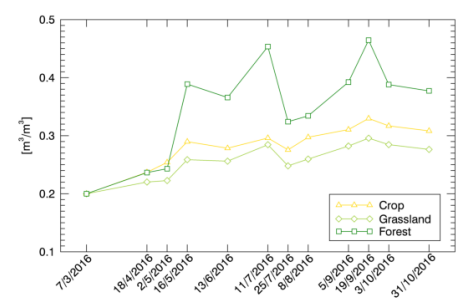

Figure 9. Kumamoto inversion results for different land cover classes. The scaling factor, appropriate for the average of the whole image, is probably too small for non-forest classes, resulting is a compressed evolution.

The average brightness of the SAR images also present a trend similar to the one in Figure 8. This can be see in Figure 10 for different land-cover classes. Here the backscatter variations, in $\mathrm{dB}$ scale, are less pronounced for the forest class.

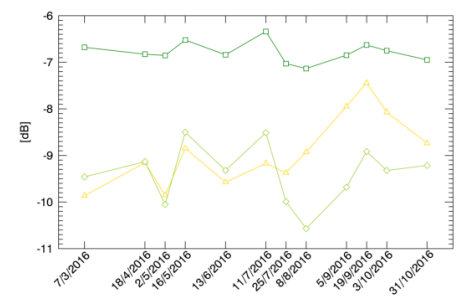

Figure 10. Kumamoto backscatter $\left(\sigma_{0}\right)$ for different land cover classes (colour coding and classes as in Figure9).

The particular high closure phase areas in Figure $5 f$ are most probably due to a rain event, as ground radar images from the Japan Meteorological Agency also show similar patterns during July, 25th. Most of the closure phases and coherence maps produced using the image of July, 25th present such pattern. On the contrary, it is not possible to recognize it from brightness images. Finally, the estimated moisture for the same date shows the signs of the rain event, as it can be seen in Figure 7.

\section{Interferometric Corrections}

The estimation of the moisture sequence allows compensating the interferometric phase and coherence on any interferogram generated with the same data set. Such corrections might be important to InSAR applications aimed at retrieving actual surface motion, to which the moisture signal is a nuisance. The interferometric phase caused by moisture variations will be in the order of a few centimeters (for L-band) and affect large areas. Fig. 11 shows the correction suggested by the forward models using the inverted moisture for an interferogram of the Kumamoto data set affected by a localized rain event. The phase variation in the scene is about $2 \mathrm{~cm}$. The coherence compensation shows that the model is not able to predict fully the coherence loss, whereas it explains well the observed closure phases. A possible explanation is that the rain event and moisture change induce an additional loss of coherence which is different in nature compared to the one modelled in conjunction with closure phases. Similar observations can be done for a second example reported here in Fig. 12.

\section{Discussion}

The performance of the inversion is influenced by different factors. The statistical noise affecting phase estimation is not the main concern here, since it is possible to average sufficiently the interferograms in space. The main issues reside with the choice of the model and model parameter mismatch.

For the inversion we have chosen a specific law $\epsilon=\epsilon(\theta)$, derived from Hallikainen et al. (1985). Deviations from the correct model will affect the inverted moisture. Our simulations show that in many cases a simple stretch of the moisture axis is enough to compensate for the model mismatch, even when the loss tangent is wrong, i.e. the ratio between the real and imaginary part of the dielectric constant. The 


\section{$25 / 7 / 2016-8 / 8 / 2016$}
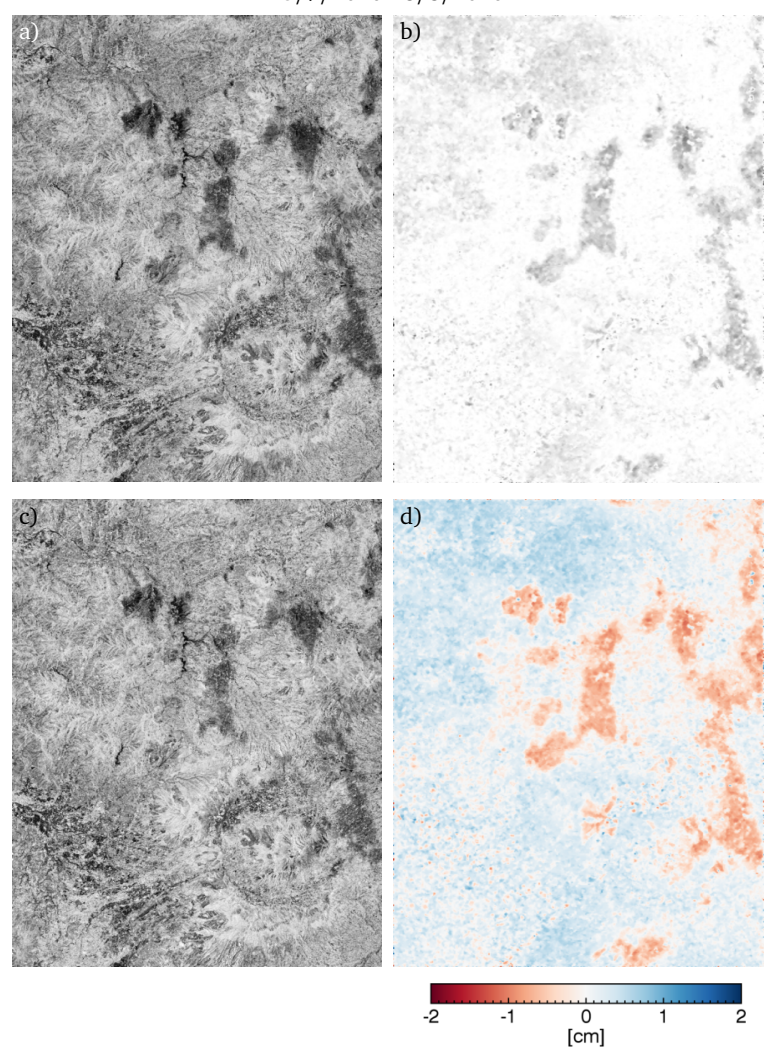

Figure 11. Original coherence (a), modeled coherence (b), compensated coherence (c) and modeled interferometric phase (d).

possibility to identify the correct dielectric law from the data is still to be investigated.

An unexpected finding is that the inversion yields consistent results over forested areas: The closure phase signal is actually very clean over forests and the inversion is stable, even if the model was developed for bare soils, and soil visibility is partially blocked over forests at L-band. A possible explanation is that the vegetation itself is contributing to the closure phase signal and the inverted moisture reflects the water status of the plants. The variation of the dielectric constant of trees has been demonstrated and studied in the past (see McDonald et al. (2002)) so that a similar model to the one proposed for soils could also apply. After all the model in De Zan et al. (2014) is built on very simple assumptions: a semi-transparent medium, scatterers at all depths, phase and amplitude dependent on the moisture level through the effect of the dielectric contant. Considering that the dielectric behaviour of soils and wood is not so different can explain why the two media can share the same interferometric modeling. Another important points con-
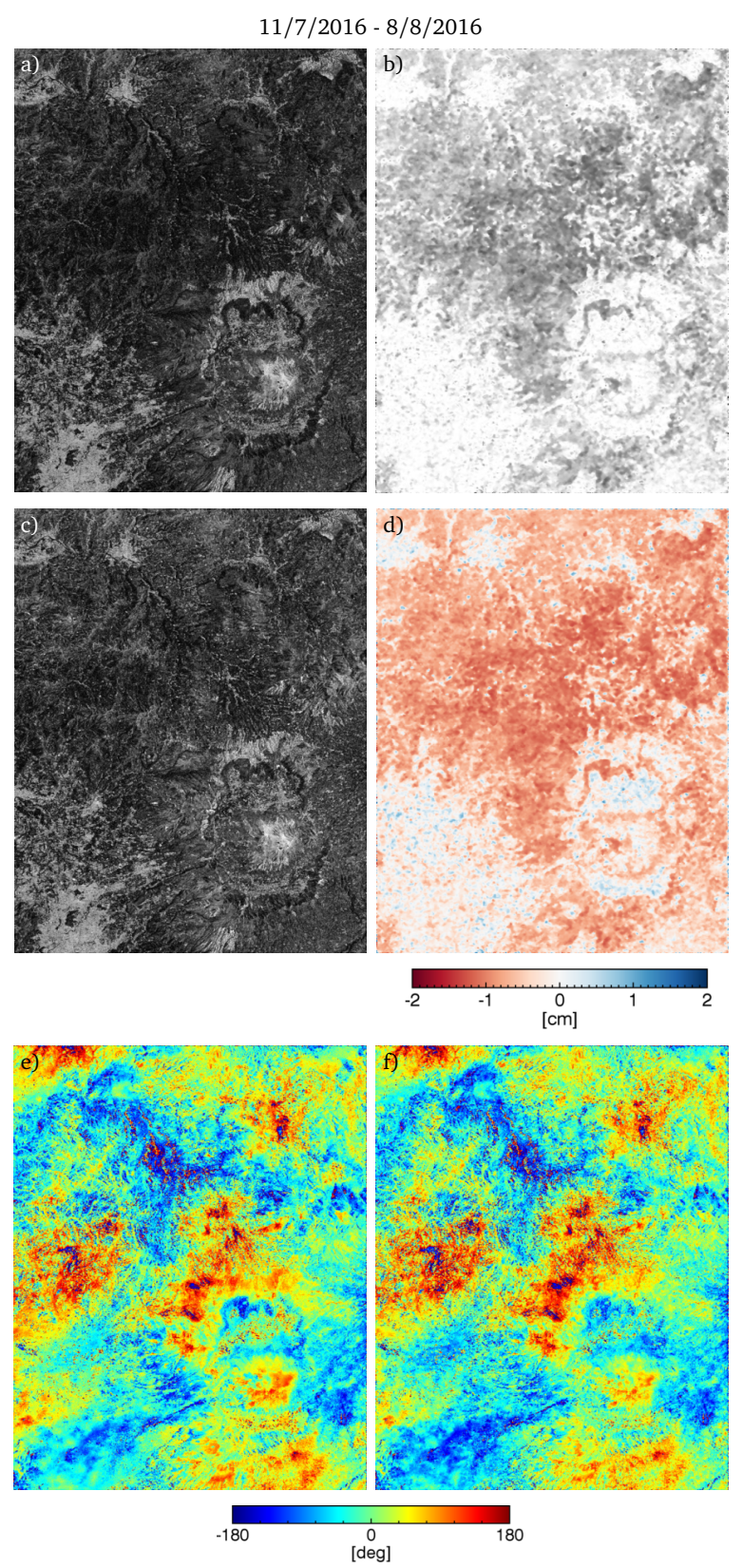

Figure 12. Original coherence (a), modeled coherence (b), compensated coherence (c), modeled interferometric phase (d), original interferogram (f) and compensated interferogram (e).

cerns the dielectric constants which are generally very large compared to air or vacuum. This implies a propagation in the denser medium (soil or wood) which is almost aligned with the normal to the surface, i.e. almost independent of the incidence angle. We expect similar effect of surfaces oriented vertically, horizontally or anything in between.

We should consider that it might be difficult to tell 
from our experiment whether closure phases are sensitive to soil or vegetation moisture, as vegetation moisture is correlated to soil moisture, possibly to soil moisture in the root zone rather than the first few centimeters of soil that L-band radar is able to sense under normal circumstances.

Indeed the closure phases measured over nonforested areas, including agricultural fields and grasslands, are typically very small (together with the inverted moisture variation). This could be an indication of an actual difference in the dynamics of moisture over fields and forests, or as an inadequacy of the model to describe both scatterers.

We notice that double-bounce scattering in the forest could even enhance the closure-phase signal observed in the data. Each bounce contributes a phase delay so that the final phase is the sum of all contributions. Assuming two bounces with equal effect on the interferometric phase, the interferogram would show a doubled phase effect, and the closure phase would also double. Fortunately the inversion is robust to a moderate increase of the closure phases. By looking at Eq. (9) one can see that a linear scaling in the moisture has a cubic effect on the closure phases: this gives some robustness to scaling in the closure phases.

If the acquisitions display a significant normal baseline variation, the interpretation of closure phases over forests would be more difficult, as volumetric effects could contribute to closure phases, beside moisture variations. In this case one should develop a method to estimate, along with the moisture contribution, also the volumetric effect. Luckily, ALOS-2 has a good orbit control and such effects are irrelevant.

It is worth mentioning that our inversion is not particularly efficient, but we have also not devoted much effort in making it so, considering that other limitations prevent from declaring it an operational tool.

\section{Conclusions}

This work has illustrated a novel method to retrieve moisture levels from SAR closure phases, overcoming the difficulties posed by ambiguities in the inverse problem. The inversion appears to work at subkilometer scale, but validation is still difficult. In general we have not found suitable ALOS-2/PALSAR-2 data sets over areas where large networks of moisture probes have been deployed. There are actually limited places in the world with stacks of coherent PALSAR-2 acquisitions. To confirm that we are observing a moisture signal we have resorted to remote sensing, in particular a good agreement was found with EUMETSAT ASCAT moisture products. Unfortunately radiometric measurements were not available on our Kumamoto test site because of radio-frequency interference.

The proposed inversion is not able to give absolute moisture values, a starting point needs to be provided. At the moment we believe that a calibration is needed also for a scaling factor, if the dielectric law is not known, since different dielectric laws will be almost equivalent to a linear transformation of the moisture axis. Whether the data themselves can tell us something about the local dielectric constant and which is the impact of a spatially varying dielectric law are still topics open for investigation. We observed different behaviours for different land cover types. Future work could also be directed at providing more efficient implementations of the algorithm and at reducing the spatial averaging to a minimum.

From the fact that the inversion works well over forests we have speculated that closure phases are sensitive to tree moisture level variations. This should be investigated more and might be interesting for forest studies. Future work should also be directed into validation with a network of moisture probes, to assess the quality of the inverse product and the need for external calibration.

Additional interesting research questions involve the possibility to include backscatter and polarimetric information in the inversion, the feasibility of the inversion for shorter wavelengths, the applicability of the inversion to different types of land cover.

Apart from all difficulties working towards a moisture product, the inversion allows a straightforward correction of interferometric phases, which is interesting for InSAR deformation applications, especially to remove long-term trends.

\section{References}

Bircher, S., Demontoux, F., Razafindratsima, S., Zakharova, E., Drusch, M., Wigneron, J.-P, \& Kerr, Y. H. (2016). L-band relative permittivity of organic soil surface layers - A new dataset of resonant cavity measurements and model evaluation. Remote Sensing, 8. doi:10.3390/rs8121024.

Brocca, L., Hasenauer, S., Lacava, T., Melone, F., Moramarco, T., Wagner, W., Dorigo, W., Matgen, P., Martínez-Fernández, J., Llorens, P. et al. (2011). Soil moisture estimation through ASCAT and AMSR-E sensors: An intercomparison and validation study across Europe. Remote Sensing of Environment, 115, 3390-3408. doi:doi:10.1016/j.rse.2011.08.003.

Crow, W. T., Berg, A. A., Cosh, M. H., Loew, A., Mohanty, B. P., Panciera, R., de Rosnay, P., Ryu, D., \& Walker, J. P. (2012). Upscaling sparse ground-based soil moisture observations for the validation of coarse-resolution satellite soil moisture products. Reviews of Geophysics, 50. doi:10.1029/2011RG000372. 
Dall, J. (2007). InSAR elevation bias caused by penetration into uniform volumes. IEEE Transactions on Geoscience and Remote Sensing, 45, 2319-2324. doi:10.1109/TGRS.2007.896613.

Das, K., \& Paul, P. K. (2015). Present status of soil moisture estimation by microwave remote sensing. Cogent Geoscience, 1, $1-21$. doi:10.1080/23312041.2015.1084669.

De Zan, F., Parizzi, A., Prats-Iraola, P., \& López-Dekker, P. (2014). A SAR interferometric model for soil moisture. IEEE Transactions on Geoscience and Remote Sensing, 52, 418-425. doi:10.1109/TGRS.2013.2241069.

De Zan, F., Zonno, M., \& López-Dekker, P. (2015). Phase inconsistencies and multiple scattering in SAR interferometry. IEEE Transactions on Geoscience and Remote Sensing, 53, 6608-6616. doi:10.1109/TGRS.2015.2444431.

Hallikainen, M. T., Ulaby, F. T., Dobson, M. C., El-rayes, M. A., \& k. Wu, L. (1985). Microwave dielectric behavior of wet soilpart 1: Empirical models and experimental observations. IEEE Transactions on Geoscience and Remote Sensing, GE-23, 25-34. doi:10.1109/TGRS.1985.289497.

Kornelsen, K. C., \& Coulibaly, P. (2013). Advances in soil moisture retrieval from synthetic aperture radar and hydrological applications. Journal of Hydrology, 476, 460 - 489. doi:10.1016/j.jhydrol.2012.10.044.

McDonald, K. C., Zimmermann, R., \& Kimball, J. S. (2002). Diurnal and spatial variation of xylem dielectric constant in Norway Spruce (Picea abies [L.] Karst.) as related to microclimate, xylem sap flow, and xylem chemistry. IEEE Transactions on Geoscience and Remote Sensing, 40, 2063-2082. doi:10.1109/TGRS.2002.803737.

Mohanty, B. P., Cosh, M. H., Lakshmi, V., \& Montzka, C. (2017). Soil moisture remote sensing: State-of-the-science. Vadose Zone Journal, 16. doi:10.2136/vzj2016.10.0105.

Morrison, K., \& Bennett, J. C. (2015). Virtual bandwidth SAR (VB-SAR) for centimetric-scale sub-surface imaging from space. International Journal of Remote Sensing, 36, 1789-1808. doi:10.1080/01431161.2015.1025922.

Morrison, K., Bennett, J. C., Nolan, M., \& Menon, R. (2011). Laboratory measurement of the DInSAR response to spatiotemporal variations in soil moisture. IEEE Transactions on Geoscience and Remote Sensing, 49, 3815-3823. doi:10.1109/TGRS.2011.2132137.

Ochsner, T. E., Cosh, M. H., Cuenca, R. H., Dorigo, W. A., Draper, C. S., Hagimoto, Y., Kerr, Y. H., Njoku, E. G., Small, E. E., Zreda, M. et al. (2013). State of the art in large-scale soil moisture monitoring. Soil Science Society of America Journal, 77, 18881919. doi:10.2136/sssaj2013.03.0093.

Peng, J., \& Loew, A. (2017). Recent advances in soil moisture estimation from remote sensing. Water, 9, $1-5$ doi:10.3390/w9070530.

Peng, J., Loew, A., Merlin, O., \& Verhoest, N. E. (2017). A review of spatial downscaling of satellite remotely sensed soil moisture. Reviews of Geophysics, 55, 341-366. doi:10.1002/2016RG000543.

Thoma, D., Moran, M., Bryant, R., Rahman, M., Collins, C. H., Keefer, T., Noriega, R., Osman, I., Skrivin, S., Tischler, M. et al. (2008). Appropriate scale of soil moisture retrieval from high resolution radar imagery for bare and minimally vegetated soils. Remote Sensing of Environment, 112, 403-414. doi:10.1016/j.rse.2007.06.021

Wagner, W., Dorigo, W., de Jeu, R., Fernandez, D., Benveniste, J., Haas, E., \& Ertl, M. (2012). Fusion of active and passive microwave observations to create an essential climate variable data record on soil moisture. ISPRS Annals of Photogrammetry, Remote Sensing and Spatial Information Sciences, 7, 315-321. doi:10.5194/isprsannals-I-7-315-2012.
Wagner, W., Lemoine, G., Borgeaud, M., \& Rott, H. (1999). A study of vegetation cover effects on ERS scatterometer data. IEEE Transactions on Geoscience and Remote Sensing, 37, 938-948. doi:10.1109/36.752212.

Wagner, W., Naeimi, V., Scipal, K., de Jeu, R., \& MartínezFernández, J. (2007). Soil moisture from operational meteorological satellites. Hydrogeology Journal, 15, 121-131.

Zwieback, S., Hensley, S., \& Hajnsek, I. (2015). Assessment of soil moisture effects on L-band radar interferometry. Remote Sensing of Environment, 164, 77-89. doi:10.1016/j.rse.2015.04.012.

Zwieback, S., Hensley, S., \& Hajnsek, I. (2017). Soil moisture estimation using differential radar interferometry: Toward separating soil moisture and displacements. IEEE Transactions on Geoscience and Remote Sensing, 55, 5069-5083. doi:10.1109/TGRS.2017.2702099. 